\title{
Chronic Constipation in Adults: The Primary Care Approach
}

\author{
A. Pali Hungin \\ Professor Emeritus of Primary Care and General Practice, Faculty of Medical Sciences, Newcastle University, Tyne, UK
}

\section{Keywords}

Chronic constipation · Management · Primary care

\begin{abstract}
Background: Primary care is normally the initial point of presentation and management of patients with chronic constipation (CC). CC has a prevalence of 3-27\%, predominantly amongst females and in the older population, and is associated with a reduced quality of life. Effective management can be a challenge without a structured approach. Summary: Whilst conventional teaching defines constipation as the number of stools per week, patients complain mainly of other symptoms such as straining and stool consistency rather than frequency. Symptoms need to be correlated with the likely causes, and ruling out secondary causes and treatment should be symptom driven, distinguishing between functional constipation and irritable bowel syndrome with CC. Treatment can be stepwise or involve combinations of therapies. There is a poor evidence base for most common managements, including lifestyle measures, although stimulant laxatives can be effective. Key Messages: Failure of routine treatment and an early consideration of alternative causes should lead to collaboration with a specialist, especially as many problems, such as pelvic floor disorders, gynaecological problems, and dyssynergic defecation, require secondary
\end{abstract}

care investigations and remedies. In light of our ageing population, CC should be considered thoughtfully and the diagnosis and management tailored to the individual, to maintain good quality of life.

(c) 2021 S. Karger AG, Basel

\section{Introduction}

Chronic constipation (CC) is an unpleasant problem which presents essentially in primary care, usually to a retail pharmacist or a general practitioner. Although it is largely managed within primary care, there are instances where specialist intervention is warranted. It is important that CC is not seen as being merely inconvenient and not worth the diagnostic effort for optimal management. A good, collaborative relationship between primary care physicians and gastroenterologists can avoid many of the misdiagnoses and pitfalls which some patients have to suffer. This is particularly important as we have an increasing, elderly population prone to constipation who need to be treated with a thoughtful diagnostic and treatment strategy. Clinicians have perhaps, in the past, been guilty of a somewhat less focussed approach to constipation, especially in older people.

Correspondence to:

A Pali Hungin, apshungin@gmail.com karger@karger.com

www.karger.com/ddi
(C) 2021 S. Karger AG, Basel

$$
\text { 는 }
$$


The aims of this study were to lay out the extent of the problem from the primary care viewpoint, to present strategies for diagnosis and management, and to underline the need for an organized and thoughtful process for optimizing patient care.

\section{The Extent of the Problem}

About $10 \%$ of all consultations in European primary care relate to digestive disorders [1], and CC is a common presenting problem. Its prevalence ranges from 3 to $27 \%$ in the general population $[2,3]$, predominantly amongst females and increasing with age. Whilst it affects $2 \%$ of the non-elderly ( $<65$ years), it affects $26 \%$ of men and $34 \%$ of women over 65 years. It has been noted that up to $75 \%$ of those in hospital care or nursing homes use laxatives [4]. This is thus a sizeable problem which contributes to discomfort and resource use.

\section{How Do Sufferers Describe Constipation?}

Conventional teaching has focussed on the number of bowel movements per week, but patients' experiences of constipation are varied and personal. The degree of straining and consistency of stools rather than the frequency seem to be predominant factors for patients. Frequency of stool has been described as "normal" by sufferers, with a wide range from 3 per day to 3 per week [4]. Stool frequency of even $<3$ per week is considered normal by many patients if there is no discomfort and/or change in frequency [4]. In a large study of people reporting constipation [5], the chief symptoms were straining (in 79\%), gas $(74 \%)$, and hard consistency (71\%), whilst infrequent bowel movement and a feeling of incomplete evacuation were reported by 57 and $54 \%$.

This wide range of symptom presentation makes it essential that each patient's problems are individually ascertained to formulate an individualized clinical response. Therapies need to be tailored to specific symptoms.

The Rome Foundation for Functional Gastrointestinal (GI) Problems has laid out definitions and guidelines for the management of functional GI disorders [6]. The Rome IV version lays out criteria for the diagnosis of functional constipation, including CC, based on symptoms such as the frequency and consistency of stools, and categorizes CC into 4 subtypes: (a) functional constipation, (b) irritable bowel syndrome with CC (IBS-C), (c) opioid-induced constipation, and (d) functional defecation disorders, including inadequate defecatory propulsion, and dyssynergic defecation [7].

\section{Categorizing Likely Causes of CC in Clinical Practice}

The first-line approach in primary care is to ascertain if the problem has a primary or secondary basis.

Primary causes are predominant and include the following:

- Functional constipation

- IBS-C

- Pelvic floor disorders

- Much less common primary causes include idiopathic megacolon or megarectum, Hirschsprung's disease, and chronic intestinal pseudo-obstruction

Secondary causes $[8,9]$ :

- Intrinsic structural problems such as colorectal cancer, diverticular disease, and rectocoele

- Metabolic and endocrine causes such as hypercalcaemia, coeliac disease, hypothyroidism, and hypercalcaemia

- Neurological problems such as spinal cord injury and Parkinson's disease

- Psychological causes such as depression, anorexia, affective disorders, and abuse history

- Medications, such as opiates, iron supplements, diuretics, and tricyclics

\section{Distinguishing between IBS-C and functional CC}

Functional constipation, essentially the chief category of CC, and IBS-C are the predominant causes of constipation seen in primary care and should be distinguished from one another.

In IBS-C:

- Abdominal pain is a feature rather than in CC

- In IBS-C, pain is often relieved by defecation and can be associated with change in frequency and form of stool

- IBS-C is characterized by abnormal stool passage (straining, incomplete evacuation, and urgency), abdominal bloating, associations with eating, and the passage of mucous

In CC:

- Bloating and discomfort rather than pain

- Hard stools

- Infrequent urge

- Straining

- Predominantly women, often first onset at puberty 


\section{Rectocoele and Enterocoele, Often Missed as a Cause of Constipation}

The management of CC should be geared to the likely causes, and amongst commonly missed problems, in females, is the presence of a rectocoele or enterocoele. A rectocoele is caused by the herniaton of rectum through rectovaginal defects, and an enterocoele is due to small intestinal pushing into the vagina. These lead to symptoms suggestive of obstructive defecation and are often associated with uterine prolapse or a cystocoele. In this situation, in addition to constipation symptoms, patients often complain of having to resort to digital vaginal manipulation to aid the passage of stool. These problems are largely related to childbirth, and a rectocoele can also be present in women otherwise seemingly healthy. Primary care physicians, trained across clinical specialities, are uniquely placed to enquire about and diagnose such conditions which can continue causing problems in the elderly and where conventional treatments have not been helpful. Effective management requires referral to a gynaecologist.

\section{CC and Risk of Cancer}

$\mathrm{CC}$ is associated with a higher prevalence of colorectal cancer and benign colorectal neoplasms, with the risks increasing with the severity of the constipation. The increase, as indicated by the incidence base ratios, has been estimated at 1.55 for cancer and 2.60 for benign tumours [10], although studies have also indicated that CC may be a short-term risk, dropping off after a year [11]. CC in itself is not considered to be the direct cause of these lesions which are likely to be mediated through longer transit times and a higher duration of mucosal contact with carcinogens such as bile acids. Nonetheless, any change in bowel habit or other alarm features alongside pre-existing CC should alert the clinician.

\section{CC: Treatment Approaches in Primary Care}

Having excluded secondary causes, the basic approach to treating CC involves either a stepwise selection or a combination of therapies and managements.

\section{Lifestyle and General Measures}

Exercise, increased fluid intake, and fibre within the normal diet have been traditionally advocated as first-line remedies for CC. However, the evidence base for these is limited - whilst recent guidelines [10] make a strong recommendation for encouraging exercise, partly for overall health benefits, the level of evidence is only moderate. For example, increased exercise can increase intestinal transit times and this does not translate into outcomes in constipation [10]. Overall lifestyle modifications do help in quality of life improvements but not with a decrease in symptom severity [12].

Equally, in patients who are not dehydrated, additional fluid intake does not seem to have a positive effect on constipation [10], and there is a low level of evidence that dietary fibre alone within the regular diet makes a difference, although this may help if taken with increased fluids.

Overall, guidelines recommend [10] that lifestyle measures may be of value in some patients to improve constipation and quality of life and contribute towards better health, but evidence linking interventions with positive outcomes is lacking.

\section{Bulking Agents and Osmotic Laxatives}

Bulking agents, in particular soluble fibre, are effective in CC and are strongly recommended in guidelines [10] even though there is relatively little evidence from large RCTs in patients with CC. They are recommended as the first-line treatment partly because of clinical experience of their use and their safety and low cost. Soluble fibre, such as psyllium, has been shown to improve the global symptoms of constipation as well as straining, defecation pain, stool consistency, and an increase in the number of weekly stools. Evidence for the use of insoluble fibre is conflicting, but on balance guidelines recommend soluble fibre over insoluble fibre [13]. However, the use of bulking agents, in particular insoluble fibre, is limited by adverse events such as bloating, distension, flatulence, and cramping pains [10].

Osmotic laxatives are commonly used in primary care. There is strong evidence for the use of polyethylene glycol, a saline laxative, which has been shown to be superior to lactulose in patients with CC, resulting in more frequent stools, looser stools, and less abdominal pain [10]. It is also associated with an increase in spontaneous complete bowl movements and can reduce the severity of straining in patients with IBS-C. Adverse effects include diarrhoea and abdominal pain. 


\section{Stimulant Laxatives}

Bisacodyl, a stimulant laxative, produces an active metabolite which stimulates and propagates contractions of the colon and enhances intestinal secretion. There is a moderate level of evidence of its effectiveness, and it is recommended in guidelines [10]. It is relatively well tolerated with diarrhoea and abdominal pain being the chief side effects. Sodium picosulphate, a locally acting stimulant laxative, similar in action to bisacodyl, can also be used and it is also well tolerated.

Senna, an anthraquinone, is commonly used by sufferers of constipation, often purchased directly from the pharmacy. However, evidence of its effectiveness is low, although it might work better when combined with a bulking agent or osmotic laxative [10].

\section{Rectal Suppositories and Enemas}

Rectal suppositories are frequently used to treat constipation, but the level of available evidence of their effectiveness is low. The most commonly used ones are overthe-counter glycerin or bisacodyl suppositories. Equally, the effectiveness of commonly used enema preparations is not supported by good evidence, but these can be used in patients in whom other measures have failed. The volume of fluid used should be carefully restricted (to 150 $\mathrm{mL}$ ) to avoid electrolyte imbalance, especially in the elderly [10].

\section{Combining Different Classes of Treatments}

Different classes of therapies can be combined and may produce a more acceptable result in an individual. The choice of laxatives should be made on an individual basis, and stool softeners, stimulants, and bulking agents can be combined [14]. Previous concerns about laxatives leading to tolerance, dependence, and addiction are almost certainly unfounded [15]. Although some people abuse laxatives, these do not cross the blood-brain barrier, so addiction is unlikely $[16,17]$.

\section{Probiotics}

These are commonly used by patients with supermarket products. However, evidence for their efficacy is generally lacking, partly due to heterogeneity in studies and the inability in identifying effective strains. Whilst some studies have shown probiotics to have beneficial effects on abdominal pain, bloating, and flatulence, in general only limited benefits were noted in studies on constipation. There is insufficient evidence to recommend a specific probiotic preparation for functional constipation [10].

\section{“Natural" and Chinese Herbal Remedies}

Many constipation sufferers prefer to use what they regard as a non-medical, natural therapy. The level of evidence available for their effectiveness is low. However, some Chinese herbal remedies have been studied in clinical trials and have shown positive results. The formulation of these products can vary, making it difficult for comparisons to be made and for specific recommendations to be offered [10].

\section{Discussion: Working with Secondary Care}

A patient with CC needs to be seen as an individual, and a good history to elucidate the likely category of constipation is important. Whilst the majority of sufferers can be managed in primary care, secondary causes, particularly structural, such as rectoceles in females, and medication-related problems, are important to rule out and to refer on if necessary. Differentiating CC from IBS$\mathrm{C}$ is important because management may vary, and in instances where the diagnosis is not clear and the management seems ineffective, specialist collaboration is needed.

Pelvic floor disorders, characterized by dyssynergic defecation, where there appears to be an obstructive picture without mechanical blockage, require secondary care investigations. Tests of bowel function and transit are not normally performed from primary care, and these may be needed to elucidate the best line of management. Colonoscopy requested directly from primary care is unlikely to be rewarding in so far as the CC is concerned.

Also important is the need to regard older patients' symptoms as being worthy of careful assessment and resist tendencies to put things down to old age. CC is a drag on quality of life, and as clinicians, we have a duty to manage it thoughtfully. 


\section{Acknowledgements}

The author acknowledges the support of the European Society for Primary Care Gastroenterology (ESPCG) and collaboration with the European Association for Gastroenterology, Endoscopy and Nutrition (EAGEN) through a series of combined presentations.

\section{Statement of Ethics}

This study is exempt from Ethics Committee approval as it is a narrative review, without the involvement of patients or clinicians as subjects.

\section{Conflict of Interest Statement}

The author is a committee member of the European Society for Primary Care Gastroenterology (ESPCG) and chair of the Rome IV Primary Care Committee, and has received advisory board funding in the last 3 years from Kyowa Kirin and Tillotts Pharmaceuticals.

\section{Funding Sources}

No funding was received for the preparation of this article.

\section{Author Contributions}

The author is the sole contributor to this article.

\section{References}

1 Seifert B, Rubin G, de Wit N, Lionis C, Hall N, Hungin $\mathrm{P}$, et al. The management of common gastrointestinal disorders in general practice a survey by the European society for primary care gastroenterology (ESPCG) in six European countries. Dig Liver Dis. 2008 Aug; 40(8):659-66.

2 Schmidt FM, Santos VL. Prevalence of constipation in the general adult population: an integrative review. J Wound Ostomy Continence Nurs. 2014 Jan-Feb;41(1):70-6; quiz E1-2.

3 Mugie SM, Benninga MA, Di Lorenzo C. Epidemiology of constipation in children and adults: a systematic review. Best Pract Res Clin Gastroenterol. 2011 Feb;25(1):3-18.

4 Schaeffer D, Cheskin L. Constipation in the elderly. Am Fam Physician. 1988;58(4):90714.

5 Johanson JF, Kralstein J. Chronic constipation: a survey of the patient perspective. Aliment Pharmacol Ther. 2007 Mar 1;25(5): 599-608.

6 Drossman DA, Hasler WL. Rome IV-functional GI disorders: disorders of gut-brain interaction. Gastroenterology. 2016 May; 150(6):1257-61.
7 Aziz I, Whitehead WE, Palsson OS, Törnblom H, Simrén M. An approach to the diagnosis and management of Rome IV functional disorders of chronic constipation. Expert Rev Gastroenterol Hepatol. 2020 Jan;14(1): 39-46.

8 Blaker P, Wilkinson M. Chronic constipation: diagnosis and current treatment options. Prescriber. 2010;21(9):30-45.

9 Chatoor D, Emmnauel A. Constipation and evacuation disorders. Best Pract Res Clin Gastroenterol. 2009;23(4):517-30.

10 Guérin A, Mody R, Fok B, Lasch KL, Zhou Z $\mathrm{Wu} \mathrm{EQ}$, et al. Risk of developing colorectal cancer and benign colorectal neoplasm in patients with chronic constipation. Aliment Pharmacol Ther. 2014 Jul;40(1):83-92.

11 Sundbøll J, Thygesen SK, Veres K, Liao D, Zhao J, Gregersen H, et al. Risk of cancer in patients with constipation. Clin Epidemiol. 2019 Apr 30;11:299-310.

12 Serra J, Pohl D, Azpiroz F, Chiarioni G, Ducrotté P, Gourcerol G, et al. European society of neurogastroenterology and motility guidelines on functional constipation in adults. Neurogastroenterol Motil. 2020 Feb;32(2): e13762.
13 Robertson G, Meshkinpour H, Vandenberg $\mathrm{K}$, James N, Cohen A, Wilson A. Effects of exercise on total and segmental colon transit. J Clin Gastroenterol. 1993 Jun;16(4):300-3.

14 American College of Gastroenterology Chronic Constipation Task Force. An evidence-based approach to the management of chronic constipation in North America. Am J Gastroenterol. 2005;100(Suppl 1):S1-4.

15 Müller-Lissner SA, Kamm MA, Scarpignato C, Wald A. Myths and misconceptions about chronic constipation. Am J Gastroenterol. 2005 Jan;100(1):232-42.

16 Ford AC, Moayyedi P, Lacy BE, Lembo AJ, Saito YA, Schiller LR, et al. American college of gastroenterology monograph on the management of irritable bowel syndrome and chronic idiopathic constipation. Am J Gastroenterol. 2014 Aug;109(Suppl 1):S2-26; quiz S27.

17 Emanuel A. Current management strategies and therapeutic targets in chronic constipation. Therap Adv Gastroenterol. 2011;4(1): 37. 